\title{
Ultrasound imaging classifications of thyroid nodules for malignancy risk stratification and clinical management: state of the art
}

\author{
Chiara Floridi ${ }^{1}$, Michaela Cellina ${ }^{2}$, Giorgio Buccimazza ${ }^{3}$, Antonio Arrichiello ${ }^{3}$, Andrea Sacrini ${ }^{1}$, \\ Francesco Arrigoni ${ }^{4}$, Giovanni Pompili ${ }^{1}$, Antonio Barile ${ }^{4}$, Gianpaolo Carrafiello ${ }^{3}$
}

${ }^{1}$ Unit of Diagnostic and Interventional Radiology, Department of Diagnostic Services, ASST Santi Paolo e Carlo, University of Milan, Milan, Italy; ${ }^{2}$ Department of Radiology, ASST Fatebenefratelli Sacco, Milan, Italy; Department of Health Sciences, Diagnostic and Interventional Radiology, San Paolo Hospital, University of Milan, Milan, Italy; ${ }^{4}$ Department of Biotechnological and Applied Clinical Sciences, University of l'Aquila, L'Aquila, Italy

Contributions: (I) Conception and design: C Floridi, M Cellina; (II) Administrative support: G Buccimazza; (III) Provision of study materials or patients: A Arrichiello; (IV) Collection and assembly of data: G Pompili, A Sacrini; (V) Data analysis and interpretation: F Arrigoni, A Barile; (VI) Manuscript writing: All authors; (VII) Final approval of manuscript: All authors.

Correspondence to: Chiara Floridi. Unit of Diagnostic and Interventional Radiology, Department of Diagnostic Services, ASST Santi Paolo e Carlo, via A. di Rudinì 8, 20142 Milan, Italy. Email: chiara.floridi@gmail.com.

\begin{abstract}
Assessing the risk of malignancy in the thyroid with ultrasound (US) is crucial in patients with nodules, as it can aid in selecting those who should have a fine-needle aspiration (FNA) biopsy performed. Many studies have examined whether the US characteristics of thyroid nodules are useful indicators of histological malignancy. Overall, these investigations have identified a few US features that are significantly more frequent in malignant thyroid nodules which can be coalesced into a defining set to be used as an indicator of a higher risk of malignancy. Despite these efforts, none of these classifications have been widely adopted worldwide, and there are still conflicting recommendations from different institutions. Understanding the role and appropriate utilization of these systems could facilitate the effective interpretation and communication of thyroid US findings among referring physicians and radiologists. In this comprehensive review, we outline the major US classification systems of thyroid nodules published in the last few years.
\end{abstract}

Keywords: Ultrasound (US); malignancy; thyroid nodule; classification

Submitted Jan 28, 2019. Accepted for publication Jun 27, 2019.

doi: 10.21037 /gs.2019.07.01

View this article at: http://dx.doi.org/10.21037/gs.2019.07.01

\section{Introduction}

A thyroid nodule is defined by the American Thyroid Association (ATA) as " a discrete lesion within the thyroid gland that is radiologically distinct from the surrounding thyroid parenchyma" (1).

Thyroid nodules are very common in the general population, and their prevalence is dependent on the identification method used, with a high prevalence found in ultrasound (US) examination, ranging from $20 \%$ to $76 \%$ in the adult population (2). Non-palpable nodules detected on
US or on other imaging examinations are called "thyroid incidentalomas" or "incidentally discovered nodules".

Thyroid nodules are more frequent in females, with an incidence 4 times higher in women than in men (3). This distribution can be explained by hormonal influences, as pregnancy is related with the increase in size and number of nodules (4). Thyroid nodules may determine gland dysfunction and compressive symptoms due to mass effect, but the main concern is to rule out their malignancy.

A US exam is a safe, non-invasive, and fast imaging 
technique: it is sufficiently sensitive for detecting thyroid nodules and identifying suspicious features and can be used to plan further investigation and management decisions $(5,6)$.

Considering that the average prevalence of thyroid nodule malignancy is variable and ranges from $4.0 \%$ to $6.5 \%$ (7-9), the accurate estimation of the risk of malignancy on US could help identify those nodules with a high risk of cancer. Moreover, many fine-needle aspirations (FNAs) may also be obviated by identifying those nodules with an acceptably low incidence of malignancy (10).

Many investigations have focused on the US characteristics of thyroid nodules as indicators of nodule malignancy, and several US systems have been developed by an array of authors and institutions to stratify the nodule malignancy risk and provide a standardized language for radiologists and endocrinologists.

Here, we offer an overview of the most recent and recognized US classification systems for thyroid nodules including the differing proposals of the Thyroid Imaging Reporting and Data System (TI-RADS) (9,11-14) along with other guidelines from renowned endocrinological and radiological associations (15-17).

\section{TI-RADS}

Many studies have investigated the feasibility of applying the breast imaging reporting and data system (BI-RADS) concept to the US evaluation of thyroid nodules for the purpose of developing a TI-RADS.

In 2009, Horvath (11) was the first to propose TIRADS as a method to stratify the estimated risk of cancer in thyroid nodules and select those nodules needing to undergo FNA.

US features considered by this classification include echogenicity, microcalcifications, shape, irregular margins, peripheral halo, and presence of suspicious lymph nodes. TI-RADS includes 10 US patterns combined into categories with the increasing risk of malignancy and nodules classified as TI-RADS score 2 to 6 (11).

The rationale of this classification system is that the risk of malignancy rises in parallel with the increase of the number of suspicious US features and with the lack of benign findings.

TI-RADS classification has been validated through a study on 502 nodules belonging to 210 patients conducted by the same Horvath (18) who demonstrated a TI-RADS sensitivity of $99.6 \%$ (95\% CI: 98.9-100.0\%), a specificity of
74.35\% (95\% CI: 68.70-80.00\%), a positive predictive value (PPV) of $82.1 \%$ (95\% CI: 78.0-86.3\%), and a negative predictive value (NPV) of $99.4 \%$ (95\% CI: $98.3-100.0 \%)$ for malignancy. The estimated risk of malignancy was $0 \%$ in TI-RADS $2,3.4 \%$ in TI-RADS 3, $10-80 \%$ in TI-RADS 4 , and $87 \%$ in TI-RADS 5.

This system has been part of standardizing thyroid US examination reports and consolidating the disparate terminologies used by radiologists and endocrinologists all over the world.

A modified version of TI-RADS that predicts malignancy risks only according to the number of suspicious US features was proposed by Kwak et al. in 2011 (9). These features include solid nodules, hypoechogenicity, microlobulated or irregular margins, microcalcifications or mixed calcification, and taller-than-wide shape.

The risk of malignancy increases as the number of suspicious US signs increases. According to this system, nodules with no suspicious US features have a $0.02-0.028$ fitted probability of malignancy, implying that it is safe to follow up with the lesion rather than perform a biopsy. Nodules in categories TI-RADS 4 or 5 with at least 1 suspicious US feature have more than 0.036 fitted probability of malignancy, indicating that a biopsy is warranted.

In a study including 167 nodules from 150 patients (19), the TI-RADS by Kwak et al. showed a sensitivity of $94 \%$, a specificity of $28.2 \%$, a PPV of $37.3 \%$, and an NPV of $91.2 \%$; meanwhile, a study on 1,293 nodules (20) showed a sensitivity of $97.4 \%$, a specificity of $29.3 \%$, a PPV of $23.3 \%$, and an NPV of $98.1 \%$.

\section{American College of Radiology (ACR)-TI-RADS}

Another version of TI-RADS has been proposed by the ACR (12) in order to identify the most clinically significant malignancies and decrease the number of FNAs on benign nodules.

This classification is not a pattern-based approach but is based on the assessment of different US features of thyroid nodules: composition, echogenicity, shape, margin, and echogenic foci; each of these features is associated with a score ranging from 0 to 3 points.

The sum of the assigned points defines the risk of malignancies according to 5 grades, with each grade corresponding to benign, minimally suspicious, moderately suspicious, or highly suspicious for malignancy. This system does not include subcategories, nor a TR 0 group to 
indicate the normal thyroid.

Indications for FNA execution or US follow-up are based on ACR-TI-RADS level and on the maximum diameter of the nodules: for risk grades TR3-TR5, a size threshold at or above which FNA should be performed is indicated. Also, the lower size limits to perform follow-up US for TR3, TR4, and TR5 nodules were established in order to reduce the number of repeat examinations for nodules that are likely to be benign or not clinically significant. Highly suspect nodules are submitted to biopsy only if they are $1 \mathrm{~cm}$ or larger, while nodules that have a low risk for malignancy should be further investigated only when they measure $2.5 \mathrm{~cm}$ or more.

The main goal of ACR TI-RADS is to balance the benefit of detecting clinically significant malignancies against the risk and cost of submitting benign nodules or indolent and non-aggressive tumours to invasive investigations and treatment. The indications for US follow-up aim at reducing the eventuality that significant lesions remain undetected over time.

In a study which included 1,112 thyroid nodules, this classification system had an area under the receiver operating characteristic curve (AUC) in discrimination of malignant nodules of 0.875 (21), while in another study on a series of 74 nodules from paediatric patients, the AUC was $0.75(22)$.

\section{European (EU)-TI-RADS}

European (EU)-TI-RADS is a classification system first proposed by Russ et al. in 2011 (23), and has been subsequently modified into an easier-to-use version, validated in a large prospective study in 2013 (24) and finally published as a European guideline in 2017 (13).

The latest version of EU-TI-RADS consists of five categories, each which is scored in correspondence to features from the US examination: EU-TI-RADS 1 refers to a US examination where no thyroid nodule is found, while the other 4 categories-nodules benign and with low, intermediate, and high risk-correspond in an increasing risk of malignancy.

EU-TI-RADS 2, with a risk of malignancy close to $0 \%$, includes two patterns: pure/anechoic cysts and entirely spongiform nodules; these nodules should be considered as benign and FNA is not indicated. EU-TI-RADS 3, with a risk of malignancy around $2-4 \%$, consists of nodules with oval shape, smooth margins, isoechoic or hyperechoic features, and a lack of any high-risk features; these nodules should be submitted to FNA only if $>20 \mathrm{~mm}$. EU TIRADS 3, with a risk of malignancy around $6-17 \%$, consists of nodules with oval shape, smooth margins, mildly hypoechoic features, and a lack of any high-risk features; the evidence of a thin halo, cystic components, comettail artefacts, peripheral vascularization, and low stiffness, lower the malignancy risk in this group, while the presence of discontinuous peripheral or rim macrocalcifications, a thick halo, predominantly central vascularization, and high stiffness, increase the risk of malignancy; the threshold for FNA is $15 \mathrm{~mm}$.

EU-TI-RADS 5 involves nodules presenting at least 1 of the following high-risk signs: non-oval shape, irregular margins, microcalcifications, marked hypoechogenicity; the malignancy rate is $26-87 \%$ and nodules must be submitted to FNA when $>1 \mathrm{~cm}$.

In addition, the authors of EU-TI-RADS noted that extrathyroidal extension with disruption of the capsular margin increases the malignancy risk of a nodule and should be described in the report. On the other hand, hyperechoic spots associated with comet-tail artefacts, along with thin halo, are suggestive of benignity and reduce the suspicion of malignancy.

This classification system also considers extra thyroid US features in stratifying the malignancy risk and the indication to FNA.

Notably, EU-TI-RADS accounts for suspicious lymphadenopathies: in case of a suspicious lymph node of thyroid origin, FNA of the lymph node itself and of the most suspicious thyroid nodule should be implemented.

In other studies, the authors of EU-TI-RADS suggest that stiffness on elastography should be considered as complementary information in nodule assessment (25), even if there is yet no reliable threshold for delineating benign from malignant nodules. They also suggest that FNA should not be indicated on a nodule if thyroid scintigraphy shows this to be warm/hot.

According to the EU-TI-RADS guidelines, the blood flow within the thyroid nodules can be investigated with different US imaging modalities, and the type of vascularization pattern can be assessed and scored from I to III: malignant nodules are usually associated with type III vascularization, while benign nodules are usually associated with type I and II signal patterns. However, EU-TI-RADS emphasizes the fact that vascularity also rises in benign nodules and increases in size, while the presence of type III vascularization shows low sensitivity, specificity, and PPV for malignancy and should be associated with other 
suspicious US findings (23). Therefore, the reliability of Color Doppler in the malignancy assessment of thyroid nodules remains controversial: the sensitivity of Doppler depends markedly on the US equipment and settings, the establishment of central vascularity is highly observerdependent, and a quantitative analysis of the Color signals is still unclear. Given this, the Color Doppler evaluation is not included in the EU-TI-RADS score, but only recommended to be used in differentiating solid tissue from thick colloid.

In a study to assess the reliability of the first version of EU-TI-RADS (26), 430 nodules were examined, and the malignancy risks of the EU-TI-RADS categories were $0 \%$ for TI-RADS 2, 2.2\% for TI-RADS 3, 5.9-57.9\% for TIRADS 4 , and $100 \%$ for TI-RADS 5.

Recently, Xu et al. (27) observed that the percentage of unnecessary FNAs based on EU-TI-RADS was 25.2\% while another study by Grani et al. (28) reported a EU-TIRADS sensitivity of $86.1 \%$, a specificity of $32 \%$, a PPV of $8.9 \%$, and an NPV of $96.7 \%$.

\section{Korean (K)-TI-RADS}

K-TI-RADS was proposed in 2016 by Shin et al. (14). In this classification system, the malignancy risk estimated by US examination is not determined by a single US sign, but by a combination of them; the rationale is that any single US predictor is not sensitive and specific enough to determine the suspicion of malignancy. Based on US patterns including solidity, echogenicity, and suspicious US characteristics, thyroid nodules in the K-TI-RADS system are classified as high suspicion, intermediate suspicion, low suspicion, and benign.

Evidence of microcalcification, speculated or microlobulated margins, and taller-than-wide shape are considered strong predictors of malignancy, with a high specificity $(>80 \%)$, while US appearance of solid internal content and hypoechogenicity is considered predictive of malignancy, with an intermediate specificity. The decision to perform FNA should be based on the calculated risk of malignancy according to the US features and nodule size, except in case of the presence of poor prognostic factors, such as suspected lymph node metastasis, extrathyroidal tumour extension, or distant metastasis from thyroid cancer.

It should be noted that nodular vascularization at Color Doppler evaluation and elastography appearance are described as potentially useful in the distinction between benign and malignant nodules, but these are not included in the classification as further investigations are needed to assess their complementary function in the risk categories.

According to a study on 2000 nodules (29), FNA criteria of K-TI-RADS categories 4 or 5 for nodules $\geq 1 \mathrm{~cm}$ and K-TI-RADS 3 for nodules $\geq 1.5 \mathrm{~cm}$, proved to have a sensitivity, specificity, PPV, NPV, and accuracy for overall malignancy of $94.5 \%, 26.8 \%, 27.5 \%, 94.3 \%$, and $42.2 \%$, respectively (30). K-TI-RADS has also been validated by a multicentre prospective study of four institutions comprising 902 thyroid nodules, which proved the significantly higher malignancy risk of solid hypoechoic nodules $(73.4 \%)$ compared to partially cystic or iso/ hyper echoic nodules $(4.3-38.5 \%$; $\mathrm{P}<0.001)$; this study also demonstrated that the malignancy risk calculated in K-TI-RADS categories 5, 4, 3, and 2 were $73.4 \%, 19.0 \%$, $3.5 \%$, and $0.0 \%$, respectively; meanwhile, the sensitivity, specificity, PPV, NPV, and accuracy of K TI-RADS for identification of malignant nodules were $95.5 \%, 58.6 \%$, $44.5 \%, 96.9 \%$, and $69.5 \%$, respectively.

\section{American Association of Clinical Endocrinologists (AACE), American College of Endocrinology (ACE), and Associazione Medici Endocrinologi (AME) classification}

AACE/ACE/AME (15) used information provided by US examination to stratify nodules into a 3-class system based on their risk of malignancy (low, intermediate, and high risk) combining US thyroid nodule features and nodule dimension with patient clinical history. FNA execution is indicated for "high US risk thyroid lesions $\geq 10 \mathrm{~mm}$ "; "intermediate US risk thyroid lesions $>20 \mathrm{~mm}$ "; "low US risk thyroid lesions only when $>20 \mathrm{~mm}$ and increasing in size or associated with a risk history and before thyroid surgery or minimally invasive ablation therapy"; "subcapsular or paratracheal lesions"; "suspicious lymph nodes or extra thyroidal spread"; "positive personal or family history of thyroid cancer"; and "coexistent suspicious clinical findings (e.g., dysphonia)".

In addition, "nodules with a major diameter $<5 \mathrm{~mm}$ should be monitored, rather than biopsied" and "in nodules with a major diameter of 5-10 $\mathrm{mm}$ that are associated with suspicious US signs, either FNA or watchful waiting may be considered".

In a prospective study on 987 thyroid nodules consecutively referred for FNA, whose aim was determining 
the diagnostic accuracy of different US classification systems and their reliability for FNA indication (31), the AACE/ ACE/AME malignancy rate was $1.1 \%$ in low-risk, $4.4 \%$ in intermediate-risk, and $54.9 \%$ in high-risk US categories of nodules. The sensitivity, specificity, PPV, NPV, and accuracy were $82 \%, 87 \%, 54 \%, 96 \%$, and $86 \%$ respectively, with a $\mathrm{k}$ coefficient of inter-observer agreement of $82 \%$, which was higher than those of other classifications. In another study including 859 FNAs (32), according to AACE/ACE/ AME guidelines, 341 (39.7\%) were low-, 489 (56.9\%) intermediate-, and 29 (3.4\%) were high-risk nodules. Of these, $88.5 \%$ and $74.9 \%$ of low- and intermediate-risk nodules, respectively, were cytologically benign, whereas $84.6 \%$ of high-risk nodules had a moderate-to-elevated risk of malignancy or were malignant. They suggested that the biopsy decision be patient-oriented (considering the clinical setting, operator expertise, patient's preference) rather than simply based on nodule size. A further study on 2,000 nodules with analysis of the diagnostic performance of different US guidelines for thyroid nodules (33), AACE/ ACE/AME classification showed a specificity of $80.4 \%$.

Despite this research, the management of nodules with intermediate risk at AACE/ACE/AME classification is still controversial. Deng et al. (34), in a study on 1,000 patients with 1,000 nodules tried to propose a solution with a risk stratification system for intermediate-risk nodules of AACE/ACE/AME classification, elaborating a predictive model: in univariate analysis, they observed that "younger patient age, smaller nodule maximum diameter, slight hypo-echogenicity, slightly ill-defined margin, no macrocalcification and indeterminate hyper-echoic spot were significantly associated with malignancy" $(\mathrm{P}<0.05)$.

With multivariate logistic regression analysis, the authors found that indeterminate hyper-echoic spot was the most significant independent risk predictor for intermediate risk, followed by slightly ill-defined margin, slight hyper-echo, and no macro-calcification; the probability of malignancy increased with the number of risk factors increasing (34).

\section{ATA classification}

The ATA classification (16) of thyroid nodules is based on echostructure, echogenicity, margins, presence/absence of microcalcifications, and shape. It provides 5-class scoring with a risk for malignancy estimated to each category in the following manner: $<1 \%$ for benign class, $<3 \%$ for very-low suspicion, $5-10 \%$ for low suspicion, $10-20 \%$ for intermediate suspicion, and $>70-90 \%$ for high suspicion.

In ATA, classifications completely exclude Color Doppler and elastosonography parameters. A previous study on 1,293 nodules by Yoon et al. (20) considered very-lowsuspicion nodules as negative, and low- to high-suspicion nodules as positive. The results demonstrated that ATA had a sensitivity of $95.3 \%$, a specificity of $37.4 \%$, a PPV of $25.2 \%$, an NPV of $97.3 \%$, and an accuracy of $47.9 \%$, with a higher specificity, $\mathrm{PPV}$, and accuracy in comparison to Kwak's TI-RADS ( $\mathrm{P}<0.001$ for all), albeit a lower specificity $(\mathrm{P}=0.024)$. In another study (19), ATA showed a sensitivity of $98.0 \%$, a specificity of $17.3 \%$, a PPV of $35.0 \%$, and an NPV of $95.0 \%$.

In the study by Persichetti et al. (31), ATA achieved a sensitivity of $81 \%$, a specificity of $87 \%$, a PPV of $54 \%$, an NPV of $96 \%$, and an accuracy of $86 \%$; the interobserver agreement was $76.9 \%$.

In the study by Grani et al. (28), the ATA classification system achieved a sensitivity of $75 \%$, a specificity of $45.3 \%$, a PPV of $9.6 \%$, and an NPV of $95.9 \%$.

In a further study including 112 children totalling 145 thyroid nodules (31), which compared radiologists' impressions and ATA risk stratification to histology and cytology results, the sensitivity in identifying malignancy was comparable between ATA guidelines (91\%) and the radiologists' impression (90\%), while the specificity of the radiologists' malignant impression (80\%) was better than the ATA guidelines (54\%).

Another study by Pandya et al., which examined 1,947 thyroid nodules (35), was performed to identify the limitations of ATA guidelines and demonstrated that PPV for cancer increased by ATA category 1 to 5 with measures of $0 \%, 2 \%, 5 \%, 12 \%$, and $28 \%$, respectively; this study stated that the ATA classification system was optimistic about cancer detection rates for the higher-risk nodules and that the inter-rater agreement among the 5 readers was fair (intraclass correlation coefficient $=0.460$ ).

\section{British Thyroid Association (BTA) US classification of thyroid nodules}

Another existing classification for the US of thyroid nodules is that of the BTA (17), which consists of five categories: normal (U1), benign (U2), equivocal/indeterminate (U3), suspicious (U4), and malignant (U5). It considers the following US features as predictors of malignancy: eccentric location of the solid portion in partially cystic nodules, non- 
smooth margins, hypoechogenicity of the solid portion, microcalcification, and taller-than-wide shape.

In a study on 104 nodules, with only U2 being classified as a negative test and only U5 being classified as a positive test, the sensitivity, specificity, positive likelihood ratio, negative likelihood ratio, PPV, NPV, and accuracy of BTA guidelines were 100\%, 91.4\%, 11.7\%, $0.0 \%, 78.6 \%, 100 \%$ and $93.5 \%$, respectively (36). In the previously cited study by Chang et al. (19), BTA demonstrated a sensitivity of $90 \%$, a specificity of $50.9 \%$, a PPV of $45.5 \%$, and an NPV of $91.8 \%$, while in the study by Persichetti et al. (31), sensitivity was $74 \%$, specificity was $92 \%$, PPV was $62 \%$, NPV was $95 \%$, and accuracy was $89 \%$. In a study on 47 patients for correctly identifying nodules requiring further investigation, BTA guidelines showed a sensitivity of $56 \%$, a specificity of $81 \%$, a PPV of $81 \%$, and an NPV of $56 \%$ (37).

\section{Comparison between the different US classifications for thyroid nodules}

It is critical to assess the risk of malignancy in the thyroid with US in order to properly identify those patients for whom FNA biopsy is a necessary priority. Many studies have investigated whether the US characteristics of thyroid nodules are useful indicators of histological malignancy. Overall, these investigations have identified a few US features that are significantly more frequent in malignant than in benign thyroid nodules, thus defining a set of US features at a higher risk of malignancy. The main features of these classifications are summarized on Table 1.

Despite these efforts, none of these classifications have been widely adopted worldwide, and there are still conflicting recommendations from several different societies. However, in the last few years, several studies have compared the different US risk stratifications systems in large cohorts of patients with thyroid nodules in order to evaluate the most accurate and useful system. The major results of studies published in the last year are listed below.

In the study of Xu et al., which included a total of 1,460 benign and 1,005 malignant nodules (27), the distribution of malignancies among ACR-TI-RADS levels was $0 \%$, $2.3 \%, 7.5 \%, 40.1 \%$, and $81.4 \%$, respectively. ACR-TIRADS showed the best sensitivity (96.6\%) in comparison to EU-TI-RADS and K-TI-RADS, but the lowest specificity (52.9\%) and AUC (0.846). K-TI-RADS had good specificity (87.4\%) but had the lowest (71.4\%) sensitivity.
In a study which included two observers and 1,055 images of thyroid nodules (38), EU-TI-RADS proved to have the higher rate of inter-observer agreement in the first set of nodules and for indication to perform FNA in comparison to the AACE/ACE/AME and ATA guidelines, in addition to ACR-TI-RADS and K-TI-RADS.

Middleton et al. (39) evaluated 3,422 thyroid nodules for which pathologic findings were available according to the ACR-TI-RADS, the K-TI-RADS, and the ATA guidelines. The rate of malignant nodules was $89.2 \%$ according to the ACR-TI-RADS, which was the best performance in comparison to the other classification systems. The percentage of benign nodules that would be biopsied was $47.1 \%$ for the ACR-TI-RADS, $79.7 \%$ for the K-TI-RADS, and $78.1 \%$ for the ATA guidelines.

In a study including 902 nodules, whose aim was to compare the diagnostic performance of ATA guidelines with K-TI-RADS and ACR-TI-RADS (40), the calculated malignancy risks for nodules of categories $5,4,3,2$, and 1 were $71.7 \%, 21.5 \%, 2.6 \%, 3.8 \%$, and $0 \%$, respectively. The ATA guidelines in this study demonstrated significantly higher diagnostic sensitivity (95.0\%) than the ACR-TIRADS $(80.2 \% ; \mathrm{P}=0.001)$, but a lower specificity $(38.1 \%$ versus $68.9 \%$; $\mathrm{P}<0.001)$; meanwhile, when compared to K-TI-RADS, the ATA guidelines showed a lower diagnostic sensitivity (100.0\%; $\mathrm{P}=0.07)$ but a higher specificity $(28.2 \%$; $\mathrm{P}<0.001)$. The unnecessary FNA rate was the lowest according to the ACR system (25.8\%), followed by the ATA $(51.2 \%)$ and $\mathrm{K}(59.4 \%)$ guidelines.

Finally, in the most recent study on 477 patients (28), Grani et al. observed that ACR-TI-RADS proved to be the most effective system when compared to other systems considered in the analysis (EU-TI-RADS, AACE/ACE/ AME, K-TI-RADS), and allowed for the largest reduction of biopsy numbers (268 of 502) with the lowest false negative rate of $2.2 \%$ (NPV, 97.8\%; 95\% CI: 95.2-99.2\%), a sensitivity of $83.3 \%$, a specificity of $56.2 \%$, and a PPV of $12.8 \%$.

Figures 1-3 summarizes some features of the different US classifications.

In conclusion, according to different studies, all US malignancy risk stratification systems demonstrated high sensitivity and specificity, but unfortunately, the results obtained are not yet sufficient to establish the best system. Prospective studies and larger cohorts are needed to standardize a US classification for application in routine clinical practice. 
Table 1 Thyroid nodule scores summary

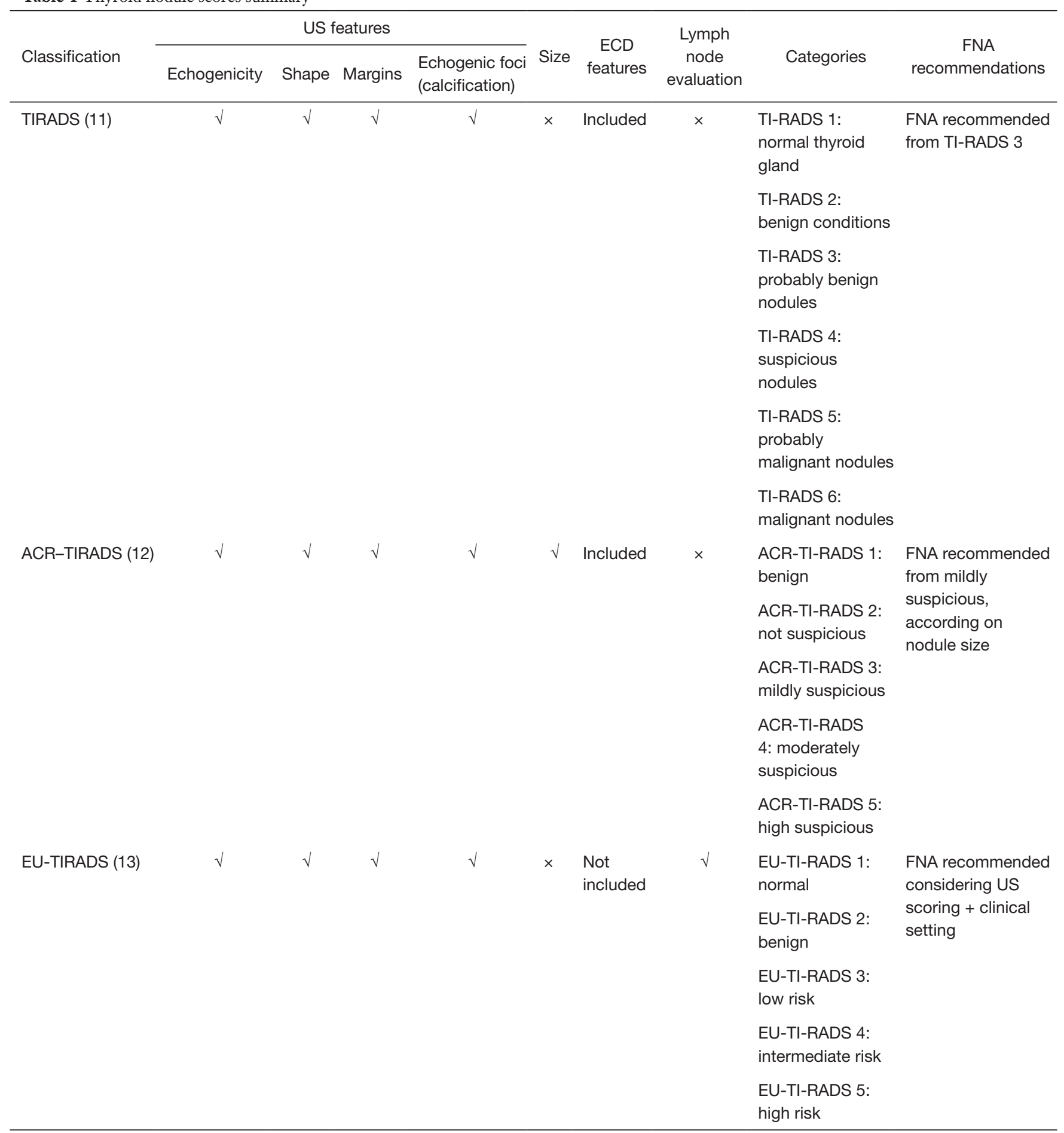

Table 1 (continued) 
Table 1 (continued)

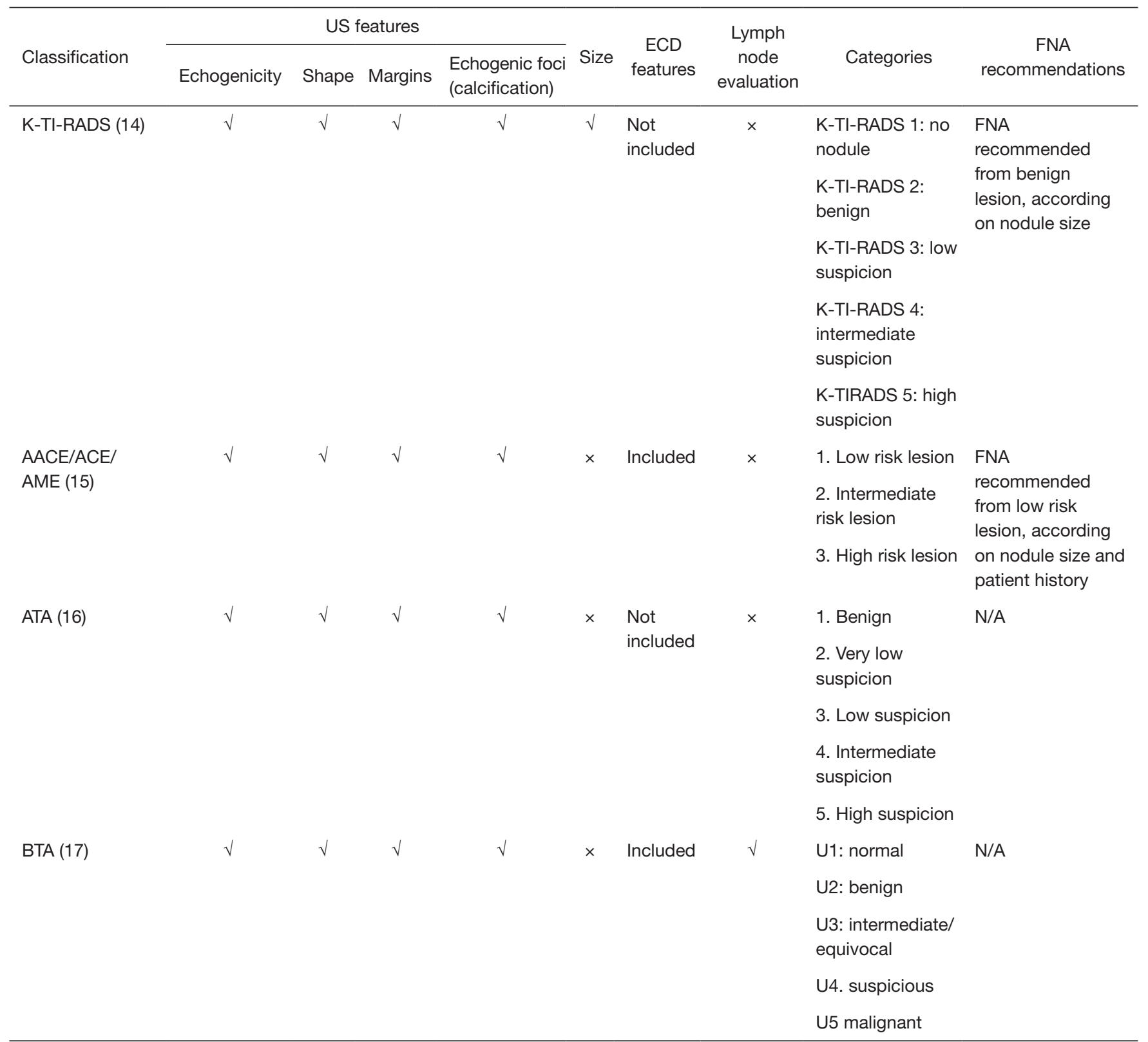

TI-RADS, Thyroid Imaging Reporting and Data System; ACR-TI-RADS, American College of Radiologists-TI-RADS; EU-TI-RADS, European-TI-RADS; K-TI-RADS, Korean-TI-RADS; AACE/ACE/AME, American Association of Clinical Endocrinologists/American College of Endocrinology/Associazione Medici Endocrinologi; ATA, American Thyroid Association; BTA, British Thyroid Association. 


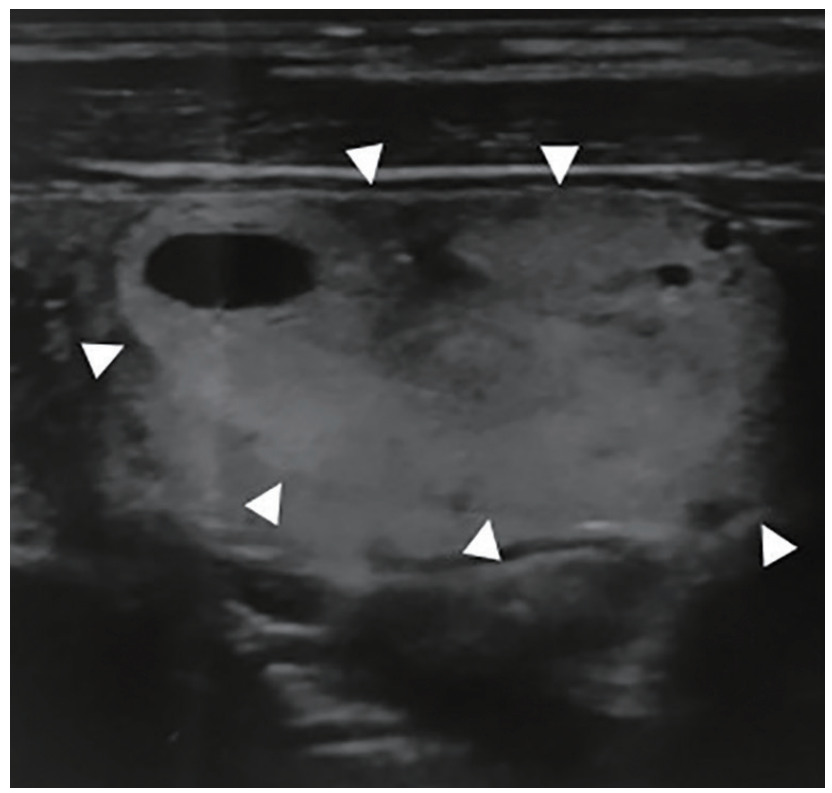

Figure 1 Transverse ultrasonography shows an isoechoic and hyperechoic mixed solid-cystic nodule with well-defined margins (arrowheads). In AACE/ACE/AME classification, this nodule is grade 1 (low-risk lesion); in TI-RADS by Horvath, the lesion is Colloid Type 1 (TI-RADS 2); in TIRADS by Kwak, it is TI-RADS 3 (not suspicious US features); in ACR-TI-RADS, it is TR 2 (not suspicious); in ATA classification, it is a nodule with low suspicion of malignancy; in EU-TI-RADS, it is grade 3 (low risk); in K-TI-RADS, it is a lesion with low suspicion of malignancy; in BTA classification, the lesion is grade U2 (benign). According to the US classifications, the patient did not undergo to fine-needle biopsy. AACE, American Association of Clinical Endocrinologists; ACE, American College of Endocrinology; AME, Associazione Medici Endocrinologi; TI-RADS, Thyroid Imaging Reporting and Data System; ACR-TI-RADS, American College of Radiology (ACR)-TI-RADS; EU-TI-RADS, European (EU)-TI-RADS; K-TI-RADS, Korean (K)-TI-RADS; BTA, British Thyroid Association; ATA, American Thyroid Association; US, ultrasound.

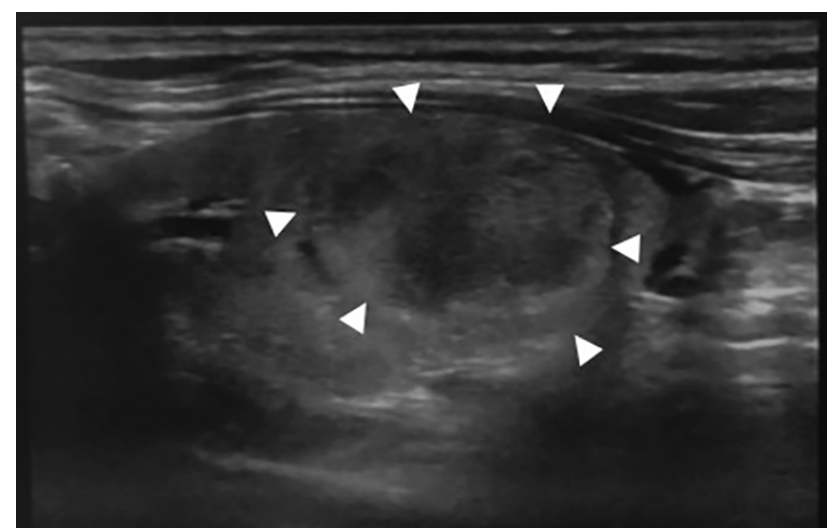

Figure 2 A longitudinal ultrasonography image shows a single, iso-hypoechoic, thyroid nodule with regular hypoechoic margins (arrowheads) and a taller-than-wide shape. In AACE/ACE/AME classification, this nodule is grade 2 (intermediate risk lesion); in TI-RADS, it is TIRADS 4a (undetermined); in TI-RADS by Kwak, it is TI-RADS 4b (2 suspicious US features); in ACR-TI-RADS, it is TR 4 (moderately suspicious); in ATA classification, the nodule has intermediate suspicion of malignancy; in EU-TI-RADS, it is grade 4 (intermediate risk); in K-TI-RADS, it is a nodule with intermediate suspicion of malignancy; in BTA classification, it is grade U3 (intermediate/equivocal). The fine-needle aspiration cytology confirmed a benign nodule. AACE, American Association of Clinical Endocrinologists; ACE, American College of Endocrinology; AME, Associazione Medici Endocrinologi; TI-RADS, Thyroid Imaging Reporting and Data System; ACR-TIRADS, American College of Radiology (ACR)-TI-RADS; EU-TI-RADS, European (EU)-TI-RADS; K-TI-RADS, Korean (K)-TI-RADS; BTA, British Thyroid Association; ATA, American Thyroid Association; US, ultrasound. 


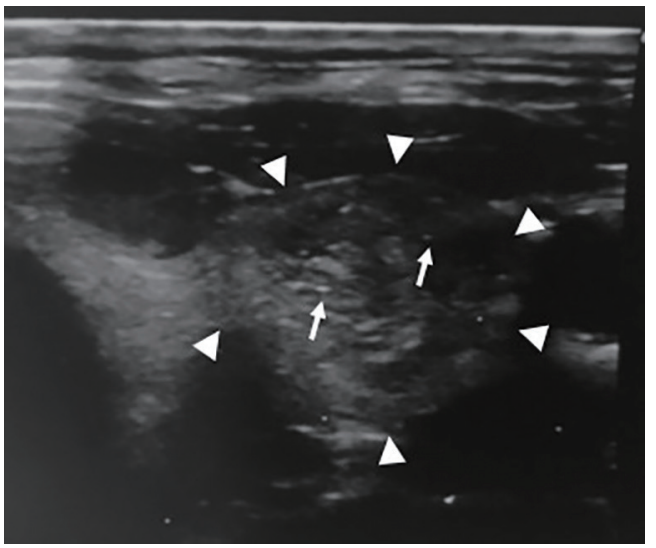

Figure 3 A transverse ultrasonography shows a single, hypoechoic, solid nodule with irregular margins (arrowheads), containing some fine areas of hyperechogenicity (arrows) without any comet-tail. In AACE/ACE/AME classification, this nodule is grade 3 (high-risk lesion); in TI-RADS by Horvath, it is TI-RADS 4a (undetermined) because there are no other nodules; in TI-RADS by Kwak, it is TI-RADS 4c (3-4 suspicious US features); in ACR-TI-RADS, it is TI-RADS 3 (mildly suspicious); in ATA classification, it is a nodule with high suspicion of malignancy; in EU-TI-RADS, it is grade 5 (high risk); in K-TIRADS, the nodule has high suspicion of malignancy; in BTA classification, it is U4 (suspicious). The fine-needle aspiration cytology confirmed a papillary carcinoma. AACE, American Association of Clinical Endocrinologists; ACE, American College of Endocrinology; AME, Associazione Medici Endocrinologi; TI-RADS, Thyroid Imaging Reporting and Data System; ACR-TI-RADS, American College of Radiology (ACR)TI-RADS; EU-TI-RADS, European (EU)-TI-RADS; K-TIRADS, Korean (K)-TI-RADS; BTA, British Thyroid Association; ATA, American Thyroid Association; US, ultrasound.

\section{Acknowledgments}

None.

\section{Footnote}

Conflicts of Interest: The authors have no conflicts of interest to declare.

Ethical Statement: The authors are accountable for all aspects of the work in ensuring that questions related to the accuracy or integrity of any part of the work are appropriately investigated and resolved.

\section{References}

1. American Thyroid Association (ATA) Guidelines Taskforce on Thyroid Nodules and Differentiated Thyroid Cancer. Cooper DS, Doherty GM, et al. Revised American Thyroid Association management guidelines for patients with thyroid nodules and differentiated thyroid cancer. Thyroid 2009;19:1167-214.

2. Periakaruppan G, Seshadri KG, Vignesh Krishna GM, et al. Correlation between Ultrasound-based TIRADS and Bethesda System for Reporting Thyroid-cytopathology: 2-year Experience at a Tertiary Care Center in India. Indian J Endocrinol Metab 2018;22:651-5.

3. Mazzaferri EL. Management of a solitary thyroid nodule. N Engl J Med 1993;328:553-9.

4. Kung AW, Chau MT, Lao TT, et al. The effect of pregnancy on thyroid nodule formation. J Clin Endocrinol Metab 2002;87:1010-4.

5. De Bernardi IC, Floridi C, Muollo A, et al. Vascular and interventional radiology radiofrequency ablation of benign thyroid nodules and recurrent thyroid cancers: literature review. Radiol Med 2014;119:512-20.

6. Barile A, Quarchioni S, Bruno F, et al. Interventional radiology of the thyroid gland: critical review and state of the art. Gland Surg 2018;7:132-46.

7. de Matos PS, Ferreira AP, Ward LS. Prevalence of papillary microcarcinoma of the thyroid in Brazilian autopsy and surgical series. Endocr Pathol 2006;17:165-73.

8. Kovács GL, Gonda G, Vadász G, et al. Epidemiology of thyroid microcarcinoma found in autopsy series conducted in areas of different iodine intake. Thyroid 2005;15:152-7.

9. Kwak JY, Han KH, Yoon JH, et al. Thyroid imaging reporting and data system for US features of nodules: a step in establishing better stratification of cancer risk. Radiology 2011;260:892-9.

10. Pompili GG, Tresoldi S, Ravelli A, et al. Use of the ultrasound-based total malignancy score in the management of thyroid nodules. Ultrasonography 2018;37:315-22.

11. Horvath E, Majlis S, Rossi R, et al. An ultrasonogram reporting system for thyroid nodules stratifying cancer risk for clinical management. J Clin Endocrinol Metab 2009;94:1748-51.

12. Tessler FN, Middleton WD, Grant EG, et al. ACR Thyroid Imaging, Reporting and Data System (TI-RADS): White Paper of the ACR TI-RADS Committee. J Am Coll 
Radiol 2017;14:587-95.

13. Russ G, Bonnema SJ, Erdogan MF, et al. European Thyroid Association Guidelines for Ultrasound Malignancy Risk Stratification of Thyroid Nodules in Adults: The EU-TIRADS. Eur Thyroid J 2017;6:225-37.

14. Shin JH, Baek JH, Chung J, et al. Ultrasonography diagnosis and imaging-based management of thyroid nodules: Revised Korean Society of Thyroid Radiology consensus statement and recommendations. Korean J Radiol 2016;17:370-95.

15. Gharib H, Papini E, Garber JR, et al. American Association of Clinical Endocrinologists, American College of Endocrinology, And Associazione Medici Endocrinologi Medical Guidelines For Clinical Practice For The Diagnosis And Management of Thyroid Nodules--2016 Update. Endocr Pract 2016;22:622-39.

16. Haugen BR, Alexander EK, Bible KC, et al. 2015 American Thyroid Association Management Guidelines for Adult Patients with Thyroid Nodules and Differentiated Thyroid Cancer: The American Thyroid Association Guidelines Task Force on Thyroid Nodules and Differentiated Thyroid Cancer. Thyroid 2016;26:1-133.

17. Perros P, Boelaert K, Colley S, et al. Guidelines for the management of thyroid cancer. Clin Endocrinol (Oxf) 2014;81 Suppl 1:1-122.

18. Horvath E, Silva CF, Majlis S, et al. Prospective validation of the ultrasound based TIRADS (Thyroid Imaging Reporting And Data System) classification: results in surgically resected thyroid nodules. Eur Radiol 2017;27:2619-28.

19. Chng CL, Tan HC, Too CW, et al. Diagnostic performance of ATA, BTA and TIRADS sonographic patterns in the prediction of malignancy in histologically proven thyroid nodules. Singapore Med J 2018;59:578-83.

20. Yoon JH, Lee HS, Kim EK, et al. Malignancy Risk Stratification of Thyroid Nodules: Comparison between the Thyroid Imaging Reporting and Data System and the 2014 American Thyroid Association Management Guidelines. Radiology 2016;278:917-24.

21. Ha SM, Ahn HS, Baek JH, et al. Validation of Three Scoring Risk-Stratification Models for Thyroid Nodules. Thyroid 2017;27:1550-7.

22. Lim-Dunham JE, Toslak IE, Reiter MP, et al. Assessment of the American College of Radiology Thyroid Imaging Reporting and Data System for Thyroid Nodule Malignancy Risk Stratification in a Pediatric Population. AJR Am J Roentgenol 2019;212:188-94.
23. Russ G, Bigorgne C, Royer B, et al. The Thyroid Imaging Reporting and Data System (TIRADS) for ultrasound of the thyroid. J Radiol 2011;92:701-13.

24. Russ G, Royer B, Bigorgne C, et al. Prospective evaluation of thyroid imaging reporting and data system on 4,550 nodules with and without elastography. Eur J Endocrinol 2013;168:649-55.

25. Cantisani V, Grazhdani H, Drakonaki E, et al. Strain US Elastography for the Characterization of Thyroid Nodules: Advantages and Limitation. Int J Endocrinol 2015;2015:908575.

26. Moifo B, Takoeta EO, Tambe J, et al. Reliability of thyroid imaging reporting and data system (TIRADS) classification in differentiating benign from malignant thyroid nodules. Open J Radiol 2013;3:103-7.

27. $\mathrm{Xu} \mathrm{T}, \mathrm{Wu} \mathrm{Y}, \mathrm{Wu} \mathrm{RX}$, et al. Validation and comparison of three newly-released Thyroid Imaging Reporting and Data Systems for cancer risk determination. Endocrine 2019;64:299-307.

28. Grani G, Lamartina L, Ascoli V, et al. Reducing the Number of Unnecessary Thyroid Biopsies While Improving Diagnostic Accuracy: Toward the "Right" TIRADS. J Clin Endocrinol Metab 2019;104:95-102.

29. Ha EJ, Moon WJ, Na DG, et al. A Multicenter Prospective Validation Study for the Korean Thyroid Imaging Reporting and Data System in Patients with Thyroid Nodules. Korean J Radiol 2016;17:811-21.

30. Creo A, Alahdab F, Al Nofal A, et al. Ultrasonography and the American Thyroid Association Ultrasound-Based Risk Stratification Tool: Utility in Pediatric and Adolescent Thyroid Nodules. Horm Res Paediatr 2018;90:93-101.

31. Persichetti A, Di Stasio E, Guglielmi R, et al. Predictive Value of Malignancy of Thyroid Nodule Ultrasound Classification Systems: A Prospective Study. J Clin Endocrinol Metab 2018;103:1359-68.

32. Negro R, Greco G, Colosimo E. Ultrasound Risk Categories for Thyroid Nodules and Cytology Results: A Single Institution's Experience after the Adoption of the 2016 Update of Medical Guidelines by the American Association of Clinical Endocrinologists and Associazione Medici Endocrinologi. J Thyroid Res 2017;2017:8135415.

33. Ha EJ, Na DG, Baek JH, et al. US Fine-Needle Aspiration Biopsy for Thyroid Malignancy: Diagnostic Performance of Seven Society Guidelines Applied to 2000 Thyroid Nodules. Radiology 2018;287:893-900.

34. Deng XH, Tang LN, Liu SQ, et al. A Proposal to Stratify the Intermediate-Risk Thyroid Nodules According to the AACE/ACE/AME Guidelines with Ultrasound Features. 
Sci Rep 2017;7:17901.

35. Pandya A, Caoili EM, Jawad-Makki F, et al. Limitations of the 2015 ATA Guidelines for Prediction of Thyroid Cancer: A Review of 1947 Consecutive Aspirations. J Clin Endocrinol Metab 2018;103:3496-502.

36. Farihah AG, Nurismah MI, Husyairi H, et al. Reliability of the ultrasound classification system of thyroid nodules in predicting malignancy. Med J Malaysia 2018;73:9-15

37. Brimioulle M, Al-Lami A, Marzouk S, et al. Ensuring patient safety when implementing a new diagnostic pathway for thyroid nodules. Ann R Coll Surg Engl 2018;100:366-70.

38. Grani G, Lamartina L, Cantisani V, et al. Interobserver agreement of various thyroid imaging reporting and data systems. Endocr Connect 2018;7:1-7.

39. Middleton WD, Teefey SA, Reading CC, et al. Comparison of Performance Characteristics of American College of Radiology TI-RADS, Korean

Cite this article as: Floridi C, Cellina M, Buccimazza G, Arrichiello A, Sacrini A, Arrigoni F, Pompili G, Barile A, Carrafiello G. Ultrasound imaging classifications of thyroid nodules for malignancy risk stratification and clinical management: state of the art. Gland Surg 2019;8(Suppl 3):S233S244. doi: 10.21037/gs.2019.07.01
Society of Thyroid Radiology TIRADS, and American Thyroid Association Guidelines. AJR Am J Roentgenol 2018;210:1148-54.

40. Ha EJ, Na DG, Moon WJ, et al. Diagnostic Performance of Ultrasound-Based Risk-Stratification Systems for Thyroid Nodules: Comparison of the 2015 American Thyroid Association Guidelines with the 2016 Korean Thyroid Association/Korean Society of Thyroid Radiology and 2017 American Congress of Radiology Guidelines. Thyroid 2018;28:1532-7. Erratum in: Correction to: Diagnostic Performance of Ultrasound-Based RiskStratification Systems for Thyroid Nodules: Comparison of the 2015 American Thyroid Association Guidelines with the 2016 Korean Thyroid Association/Korean Society of Thyroid Radiology and 2017 American College of Radiology Guidelines Ha EJ, Na DG, Moon W-J, Lee YH, and Choi N Thyroid 2018;28:1532-7. DOI: 10.1089/ thy.2018.0094. [Thyroid. 2019]. 